\title{
NEUROBLASTOMA: AN UNUSUAL PRESENTATION
}

S. N. Pathak ${ }^{1}$, Sanchari Ray ${ }^{2}$

\section{HOW TO CITE THIS ARTICLE:}

SN Pathak, Sanchari Ray. "Neuroblastoma: an unusual presentation". Journal of Evolution of Medical and Dental Sciences 2013; Vol. 2, Issue 46, November 18; Page: 8967-8971.

ABSTRACT: Neuroblastoma is a childhood malignancy derived from the primordial neural crest cells that form the sympathetic nervous system. These tumours are notorious for their early tendency of metastasis. Symptoms arising from these such as proptosis can be the only manifestation of metastatic neuroblastoma [1]. Early investigation is important, as metastatic disease requires aggressive management. We present a case of a 6 year old girl initially presenting with proptosis.

INTRODUCTION: Virchow originally described Neuroblastoma in 1863 . By the beginning of the $20^{\text {th }}$ century, its origin in sympathetic tissue was determined by Zuckerkandl and Kohn. It is the third most common pediatric malignancy, after leukaemia and central nervous system tumours [2]. The median age at diagnosis is 22 months, and more than $95 \%$ cases are diagnosed by the age of 10 years. Approximately $1 \%$ of the patients present initially evidence of metastatic disease without a readily identifiable primary lesion. Neuroblastoma commonly metathesizes to bone $(60 \%)$, regional lymph nodes (45\%), orbits (20\%), liver (15\%), intracranial (14\%) and lung (10\%)[2].

CASE REPORT: A 6 year old girl presented with the complaint of progressively increasing proptosis of bilateral eyes. The girl underwent contrast enhanced CT Scan of brain and orbits which revealed:

1. Bilateral enhancing retro-orbital masses.

2. Enhancing soft tissue masses overlying the bilateral parietal bones.

3. Separation of the coronal sutures.

4. Enhancing extra axial intracranial soft tissue mass suggestive of dural metastasis.
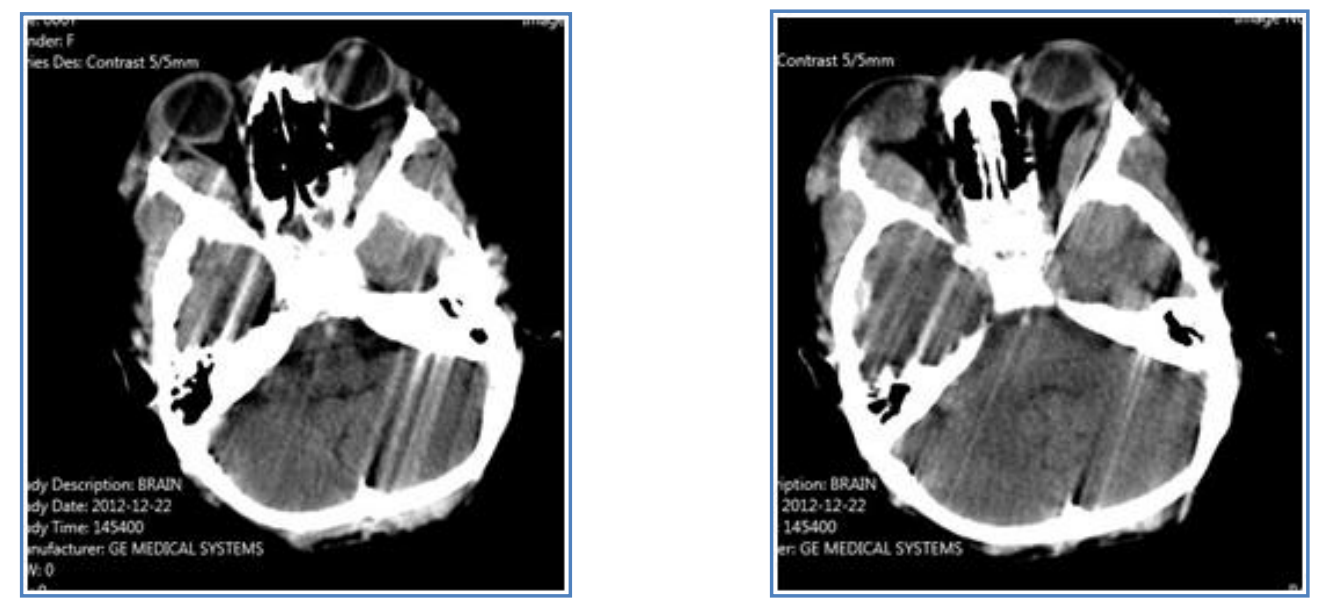

Fig. 1: Bilateral enhancing retro-orbital masses. 


\section{CASE REPORT}
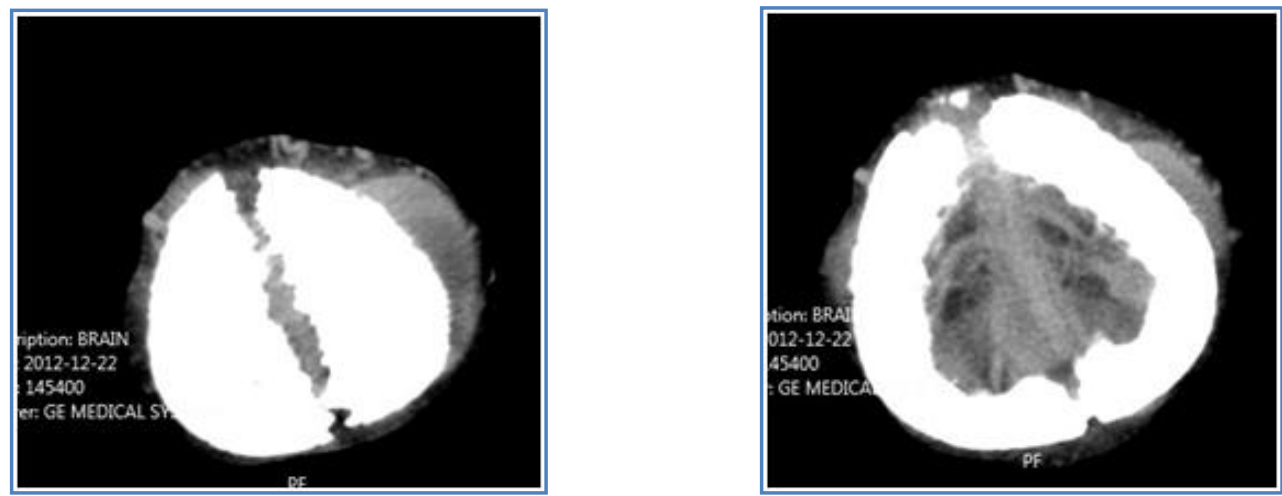

Fig. 2: Enhancing soft tissue masses overlying the bilateral parietal bones
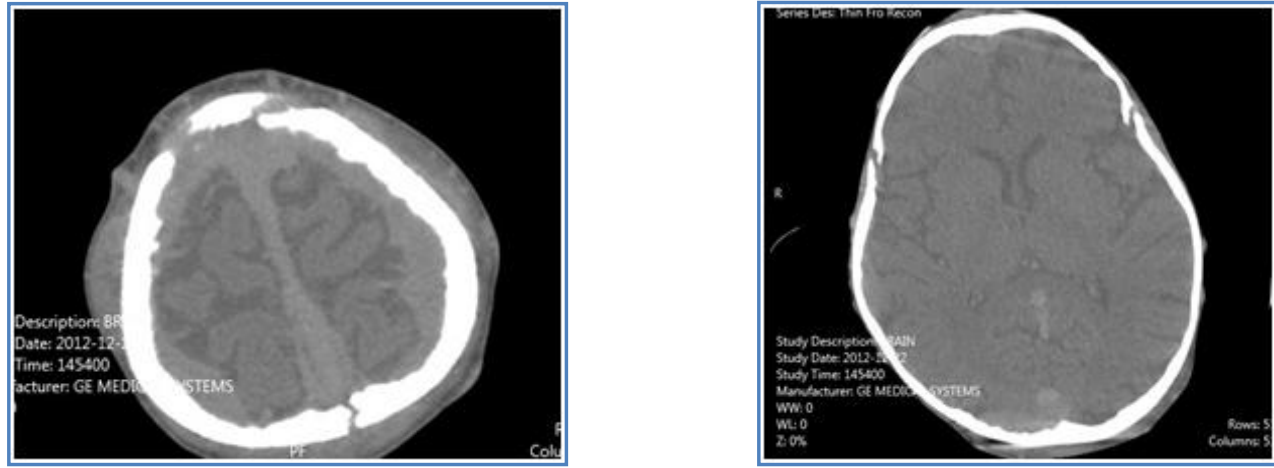

Fig. 3: Separation of the coronal sutures.
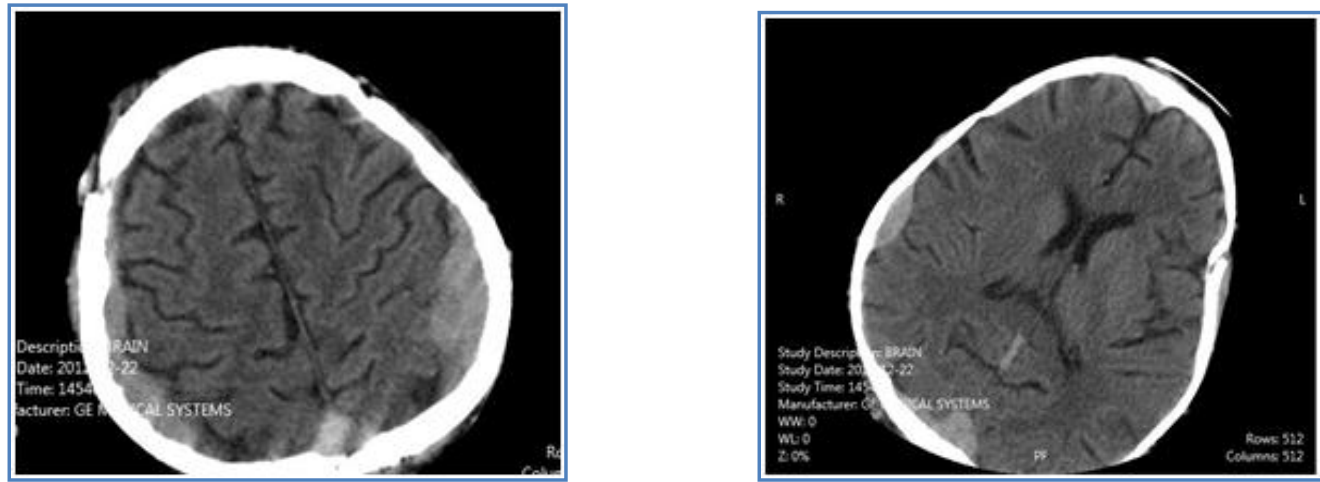

Fig. 4: Enhancing dural metastasis.

The patient subsequently underwent an abdominal ultrasound which revealed a large rounded mixed echoic mass in the left paraspinal region with multiple calcific foci causing obstructive hydronephrosis of the left kidney. 


\section{CASE REPORT}

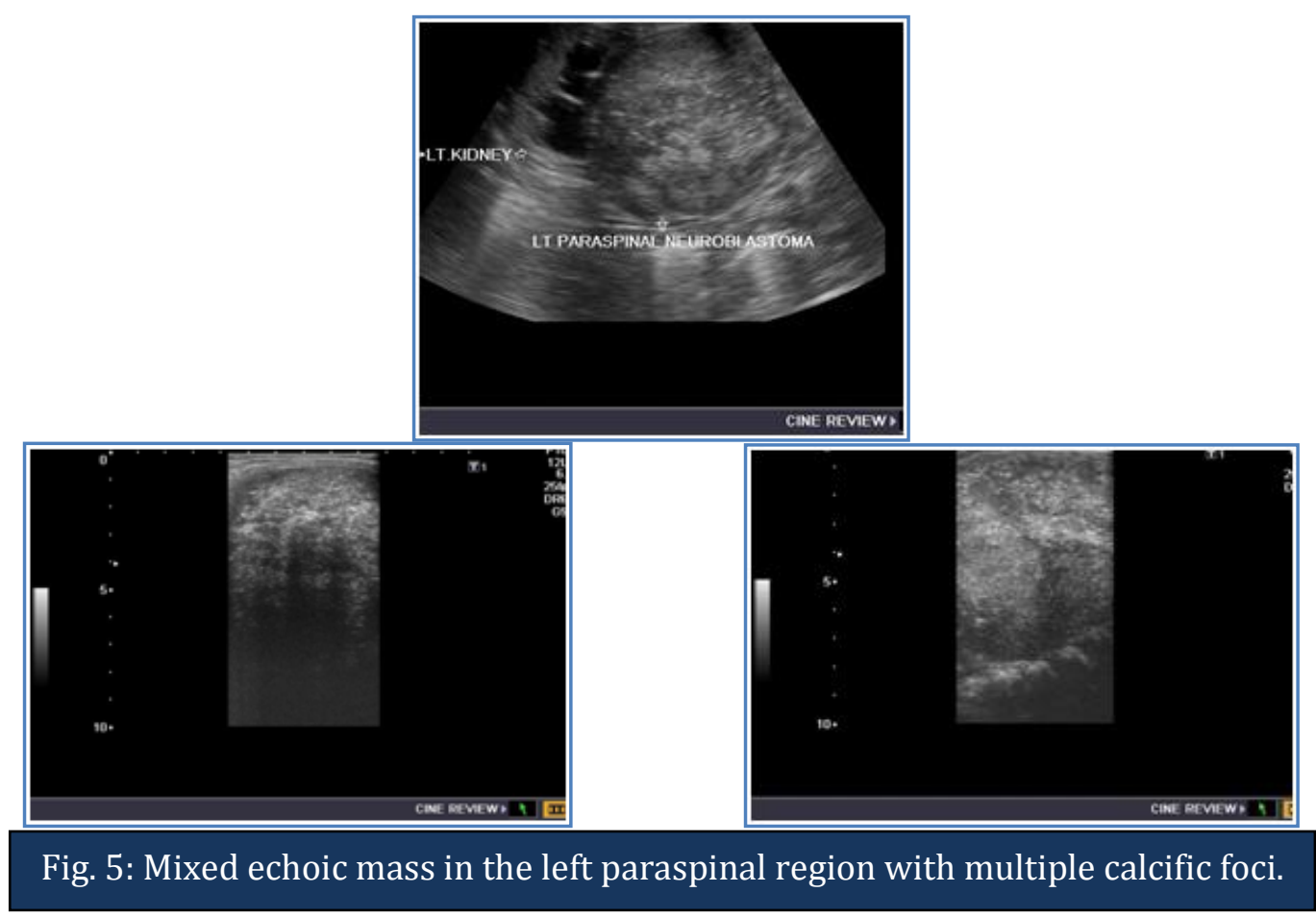

USG guided FNAC was done and the mass proved to be neuroblastoma and chemotherapy was planned. The patient responded well with regression of symptoms.

DISCUSSION: Neuroblastoma is a childhood malignancy with high incidence of metastatic disease at presentation. Approximately $20 \%$ of the cases have orbital metastasis. The primary tumour can occur anywhere from sympathetic tissue. The most common sites of origin are adrenal medulla (35\%), extra adrenal retroperitoneum (30-35\%) and posterior mediastinum (20\%). Less common sites are the neck (1-5\%) and pelvis (2-3\%). Unusual locations such as the thymus, lung, kidney, anterior mediastinum, stomach \&cauda equina have also been described[2].

Although rare, there are documented cases of familial neuroblastoma and may occur in patients with other disorders including Von-Recklinghausen disease, Beckwith - Wiedemann syndrome, Di George Syndrome etc.

Patients may present with abdominal distention, malaise, weight loss, Horner Syndrome and Opsoclonus-Myoclonus. Two-third of the patients may have metastatic bone disease at presentation, when this manifests as limping \& irritability, it is known as Hutchinson Syndrome.

The radiological appearance of these tumours are varied according to the location.

Plain radiographs may show a posterior mediastinal, retroperitoneal or neck mass. Metastatic disease may be radiologically manifested as focal lucent/sclerotic lesion in bones, pedicle erosion from intraspinal extension, cranial suture widening (from dural metastasis).

Ultrasound may reveal heterogenously echogenic masses with calcifications. It also aids in the evaluation of other organs such as liver for the evidence of metastasis.

Contrast enhanced CT scan is the most commonly used imaging modality for assessment of neuroblastoma. It reveals extent of tumour, organ of origin, regional invasion, vascular encasement, adenopathy \& calcification and distant metastasis. A common intracranial metastatic pattern is dural 
disease which may appear as enhancing meninges and meningeal cakes and masses. These may cause regional mass effect on underlying brain tissue and may extend into the sutures, resulting in sutural widening.

Because of excellent tissue discrimination \& multiplanar imaging, MR is superior to CT for determination of organ of origin \& regional invasion. MR is the preferred modality for intraspinal extension of primary tumour \& for bone morrow disease. The primary tumour as well as the metastasis enhances strongly on administration of contrast.

Bone scan using technetium 99m methylene diphosphonate is more sensitive for detection of bony metastasis than other methods.

(18)F-FDG is superior in depicting stage 1 and 2 neuroblastoma, although (123)I-MIBG may be needed to exclude higher-stage disease. (18)F-FDG also provides important information for patients with tumors that weakly accumulate (123)I-MIBG and at major decision points during therapy (i.e., before stem cell transplantation or before surgery). (18)F-FDG can also better delineate disease extent in the chest, abdomen, and pelvis. (123)I-MIBG is overall superior in the evaluation of stage 4 neuroblastoma, especially during initial chemotherapy, primarily because of the better detection of bone or marrow metastases [3].

Imaging differential diagnosis in this case

1. Spinal ganglioneuroma

- Often cannot be differentiated from neuroblastoma based on imaging characteristics.

- Metastases usually indicate neuroblastoma

- compared with ganglioneuromas, tumour calcification in neuroblastomas is more often amorphous and of a rough pattern[4].

- Calcification in ganglioneuroma is typically fine and speckled but may be coarse and it shows slight to moderate enhancement which may be heterogeneous or homogeneous[4].

2. Spinal schwannoma and neurofibroma

- centered on neural foramen

- primarily seen in middle-aged adults

CONCLUSION: Proptosis in children can be the only manifestation of metastatic neuroblastoma. Early investigation is important as metastatic disease requires aggressive management with multiagent chemotherapy. A multimodality approach is required for the thorough assessment of the behavior of the primary tumour and the metastatic disease.

\section{REFERENCES:}

1. ST Hlongwane, M Pienaar, G. Dekker, A Brandt, D Van der Merwe, H B Louw, S Dajee : Proptosis as a manifestation of neuroblastoma. SA Journal of radiology: December 2006:31-32.

2. Gael J. Lonergan, Cornelia M. Schwab, Eric S. Suarez, Christian L. Carlson: Neuroblastoma, ganglioneuroblastoma and ganglioneuroma: Radiologic-Pathologic correlation; Radiographics: july $2002,22,911-934$. 
3. Sharp SE, Shulkin BL, Gelfand MJ, Salisbury S, Furman WL:123I-MIBG scintigraphy and 18FFDG PET in neuroblastoma: journal of nuclear medicine2009 Aug;50(8):1237-43.

4. Dr Jeremy Jones and Dr Sara et al http://radiopaedia.org/articles/paraspinal-ganglioneuroma.

\section{AUTHORS:}

1. S.N. Pathak

2. Sanchari Ray

\section{PARTICULARS OF CONTRIBUTORS:}

1. Assistant Professor, Department of Radiology, Patna Medical College \& Hospital, Patna.

2. Junior Resident, Department of Radiology, Patna Medical College \& Hospital, Patna.

\section{NAME ADDRESS EMAIL ID OF THE} CORRESPONDING AUTHOR:

Dr. S.N. Pathak,

301-C, Nandan Tower,

Colony More, Kankarbagh,

Patna, Bihar - 800020.

Email - snpathak301@gmail.com

Date of Submission: 21/10/2013.

Date of Peer Review: 22/10/2013.

Date of Acceptance: 07/11/2013.

Date of Publishing: 13/11/2013 